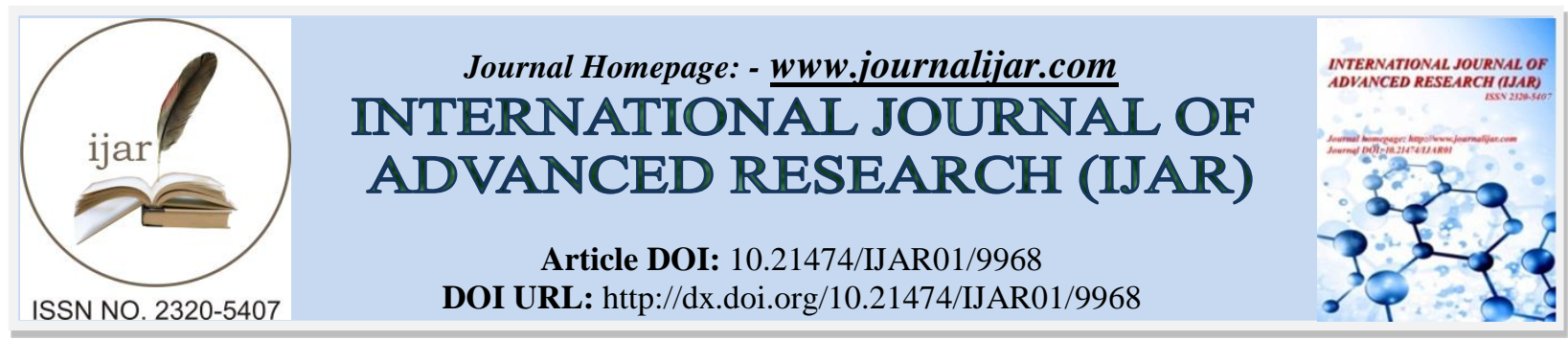

RESEARCH ARTICLE

\title{
MEATOPLASTIE URETRALE POUR UNE BEANCE IATROGENE CHEZ UNE PATIENTE PRESENTANT UN HEMATOCOLPOS. (A PROPOS D'UNE OBSERVATION).
}

\author{
Moulay Elmehdi EI Hassani ${ }^{1}$, Saad Benali ${ }^{1}$, Abdellah Babahabib ${ }^{1}$, Jaouad Kouach ${ }^{1}$, Mohammed Ghadouane ${ }^{2}$ \\ and Driss Rahali Moussaoui ${ }^{1}$. \\ 1. Service de Gynécologie et Obstétrique Hôpital Militaire d'Instruction Mohammed V - Rabat. \\ 2. Service d'Urologie, Hôpital Militaire d'Instruction Mohammed V - Rabat.
}

\section{Manuscript Info}

\section{Manuscript History}

Received: 05 September 2019

Final Accepted: 07 October 2019

Published: November 2019

Key words:-

hematocolpos; gap of the urethral meatus; magnetic resonance imaging; vaginoplastie; meatoplasty.

\begin{abstract}
Hematocolpos is the menstrual blood retention in the vagina at puberty. It is easy to diagnose and to take care of, when it is due to an imperforation of the hymen; however, when it is secondary to a vaginal malformation, like partial atresia or diaphragm. MRI is essential for diagnosis, it allows to search for other genito-urinary malformations and to guide surgical procedure. This surgery must be performed by a specialized team, to prevent recurrence and iatrogenic complications, which can induce sexual and fertility disorders in the future.

The autors relate a case of hematocolpos, which is secondary to an atresic of the lower third vagina, diagnosed in a 14 years old girl.

This observation is an example of a lack of knowledge of the diagnosis and wrong first management, complicated by iatrogenic urinary incontinence.

The diagnosis of the hematocolpos and the vaginal malformation was made by the MRI. The treatment consisted on: hematocolpos evacuation associated with a vaginoplasty and urethral meatoplasty.
\end{abstract}

Copy Right, IJAR, 2019,. All rights reserved.

\section{Introduction:}

L'hématocolpos est défini par une rétention vaginale de la menstruation. Il se constitue à la puberté dès la première menstruation. L'imperforation hyménéale est l'étiologie la plus fréquente. Un diaphragme vaginal complet plus haut situé, ou une atrésie vaginale partielle sont des causes plus rares. L'endomètre est en général fonctionnel [1,2,3].

Il se traduit sur le plan clinique par des douleurs pelviennes cycliques et une aménorrhée primaire. Plus rarement, il peut se révéler par une masse pelvienne.

L'échographie est l'examen de choix pour le diagnostic de l'hématocolpos. L'imagerie par résonance magnétique (IRM) est l'examen d'imagerie de référence pour confirmer l'hématolcolpos et exclure d'autres malformations du canal de Müller ou des malformations urologiques associées.

L'hyménéotomie est le traitement de l'hématocolpos par imperforation hyménéale. Le traitement de la malformation causale est plus complexe pour les hématocolpos révélateurs d'une malformation.

Corresponding Author:-Moulay Elmehdi El Hassani.

Address:-Service de gynécologie et obstétrique Hôpital militaire d'instruction Mohammed V - Rabat. 


\section{Observation:-}

Il s'agit d'une jeune fille âgée de 14ans, fille unique d'une fratrie de trois, sans antécédent pathologique particulier. Elle avait consultait deux mois auparavant dans une autre structure pour aménorrhée primaire associée à des douleurs pelviennes cycliques, une pollakiurie ainsi que des épreintes et ténesmes.

Sa maman avait eu une puberté normale et il n'y avait pas de cas similaires ni de pathologie génétique dans la famille. A l'interrogatoire, la mère rapportait que sa grossesse s'était déroulée normalement avec un accouchement par voie basse d'un poids normal et une croissance sans anomalie.

\section{L'échographie avait montré un hématocolpos avec hématométrie (Figure 1)}

La jeune fille avait bénéficié d'une tentative de dilatation par voie basse de ce qui semblait être l'orifice vaginal (qui n'était en fait que le méat urétrale), devant l'échec le chirurgien avait réalisé un drainage de l'hématocolpos par hystérotomie postérieur, par voie abdominale. L'évolution était marquée par l'amélioration partielle de sa symptomatologie avec persistance de l'aménorrhée et apparition de signes urinaires à type de fuites urinaires spontanées et d'énurésie, qui n'existaient pas auparavant.

La patiente était adressée alors dans notre formation pour prise en charge. L'examen clinique a découvert un morphotype normal, taille de 1 mètre $55 \mathrm{~cm}$ pour un poids de $45 \mathrm{~kg}$. Le stade de maturation pubertaire correspondait au stade 4 de Tanner. Il n'y avait pas de signes d'hyperandrogénie. La pilosité et les glandes mammaires étaient bien développées. Au niveau vulvaire, les grandes et petites lèvres étaient bien développées, le clitoris était de taille normale, le méat urétral était visible mais béant avec une fuite urinaire à l'effort. Par ailleurs, il n'y avait pas d'orifice vaginal. Le toucher rectal permettait de percevoir à travers la cloison recto-vaginale une masse rénitente haut située et douloureuse.

Une IRM pelvienne était alors demandée objectivant une rétention intra-vaginale et intra-utérine sans anomalies morphologiques de l'utérus, avec une atrésie du tiers inférieur du vagin. Les ovaires et les reins étaient en place et de taille normale [Figure 2, 3, 4, 5].

La patiente était alors programmée pour plastie vaginale et correction de la béance urétrale. Le geste était réalisé sous rachianesthésie. Le premier temps de notre intervention était vulvaire. La mise en place d'une sonde vésicale avait révélé une fuite urinaire en dehors de la sonde (Figure 6). Une incision arciforme à convexité antérieure avait créé un pertuis entre l'urètre, la vessie en avant et le rectum en arrière. Un décollement vésico-rectal délicat était réalisé sur plus de deux centimètres sans pouvoir atteindre l'hématocolpos. Devant le risque de lésion rectale et vésico-urétrale, un deuxième temps pelvien était nécessaire. Une reprise de l'incision Pfannenstiel était réalisée.

L'exploration de la cavité pelvienne a mis en évidence un utérus et des annexes d'aspect normal. L'hématocolpos bombait dans le Douglas. L'ouverture du péritoine postérieur en dessous de l'insertion des utéro-sacrés (Figure 7), avait facilité l'ouverture du vagin postérieur et permit le drainage de $300 \mathrm{cc}$ de sang digéré. Après une toilette pelvienne et vaginale, une bougie de Hégard $\mathrm{n}^{\circ} 12$ était introduite par l'orifice vaginal supérieur permettant l'orientation de la dissection par voie basse et l'ouverture en bas de la cavité vaginale. Ainsi la paroi vaginale était décollée au niveau de son bord inférieur et ramenée jusqu'à l'orifice vulvaire au dessous du méat urétral puis fixée par des points simples au fil résorbable 2/0 (Figure 8). L'intervention était terminée par une réfection du méat urétral, par la mise en place d'un point au dessus et deux points en dessous de la sonde vésicale avec un fil 5/0 résorbable (Figure 9). La sonde vésicale était gardée 10 jours et une dilatation par bougie de Hégar $\mathrm{N}^{\circ} 12$ était faite quotidiennement à partir du troisième jour pour assouplir le néo-orifice vaginal (Figure 10).

Les suites postopératoires étaient simples, marquées notamment par la disparition des fuites urinaires ainsi que de l'énurésie après ablation de la sonde vésicale. Après cicatrisation, le méat urétral n'était plus béant et l'orifice vaginal laissait passer facilement une bougie de $12 \mathrm{~mm}$ de diamètre (Figure 11).

Après 25 jours la patiente avait eu ses menstruations. Le contrôle après trois et six mois trouvait un orifice vulvaire perméable, et la patiente avait un cycle menstruel normal. 


\section{Discussion:-}

La puberté reste la période la plus fréquente de la découverte d'un hématocolpos. Le développement normal des caractères sexuels secondaires coexiste avec une aménorrhée primaire. Le diagnostic d'absence d'utérus risque d'être porté à tort [4]. Des douleurs pelviennes cycliques vont révéler l'hématocolpos. Parfois, les douleurs sont abdominales [5] ou pseudo-appendiculaires [6]. Des douleurs lombaires [7], ou à type de sciatalgies avec déficit sensitif et moteur du membre inférieur [8] ont été rencontrées. Les signes urinaires dus à une compression peuvent être à l'origine d'une rétention aiguë [9, 10, 11, 12, 13], d'une dysurie ou d'une hydronéphrose bilatérale [14]. Dans notre observation, la symptomatologie était dominée par les fuites urinaires; causées par une béance iatrogène du méat urétral, dilaté lors de la première intervention, car pris à tort pour orifice vulvaire. Les complications sont à type d'endométriose pelvienne [15], d'infection, d'adénose vaginale [16, 17] et d'infertilité. Un cas de carcinome vaginal a été rapporté dans la littérature [18].

Parmi les malformations associées ont été décrites: syndactylie [19], imperforation du canal anal [20]. Tran [2] conseille la recherche de malformations associées génito-urinaires et ano-rectales. La cryptoménorrhée douloureuse par imperforation hyménéale para-pubertaire avec aménorrhée primaire et douleurs pelviennes est le tableau le plus typique. Le bombement à la toux est caractéristique.

L'hématocolpos est plus rarement découvert derrière un septum vaginal transverse obturant ou une agénésie vaginale partielle. Dans un cas l'examen histologique a montré la présence d'éléments mésodermiques mülleriens [19]. L'hématocolpos d'un hémi-vagin borgne avec duplication génitale complète ou partielle s'accompagne toujours ou presque d'une agénésie urinaire homolatérale (22 filles sur 24 porteuses de duplications avaient une agénésie, une dysplasie ou une hypoplasie rénale homolatérale [21]). L'aplasie rénale est l'anomalie la plus souvent décrite: 120 cas mondiaux avec $10 \%$ de complications urologiques (infections urinaires, rétentions, etc.) ont été rapportés. Une fistule postopératoire d'un uretère ectopique a révélé l'agénésie partielle de l'arbre urinaire [22].

Le diagnostic de l'imperforation hyménéale est possible in utero par l'échographie.

Trois cas d'hydrométrocolpos ont été retrouvés dans la littérature [23-25]. La découverte d'un hydrométrocolpos in utero a l'intérêt de faire rechercher des associations malformatives rénales.

Les examens d'imagerie sont importants pour redresser le diagnostic en cas de doute diagnostique. L'échographie sus-pubienne montre une image rétrovésicale finement échogène. Cette collection est surmontée par la cavité utérine communicante, qui est souvent de petite taille, dilatée par un contenu liquidien en cas d'hématométrie [4]. Un hématosalpinx ou un épanchement péritonéal peuvent être trouvé [2]. L'examen échographique analyse aussi les malformations utérines. L'agénésie rénale est systématiquement recherchée surtout en cas de duplication génitale [1]. L'échographie peut montrer aussi une dilatation urétéro-pyélocalicielle en cas de compression des vois urinaires par l'hématocolpos [3]. L'IRM a comme l'échographie l'avantage de l'innocuité chez une jeune fille. Elle serait la meilleure technique d'exploration complémentaire donnant sur les séquences pondérées en T2 une très bonne analyse anatomique morphologique de la malformation et permettant la recherche d'autres malformations génitourinaires. Les séquences pondérées en T1 confirmeront le contenu hématique dans le vagin et dans la corne utérine sus-jacente $[4,5]$. Le traitement de l'hématocolpos est toujours chirurgical et est fonction de la malformation causale. L’imperforation hyménéale nécessite une hyménéotomie. Les malformations vaginales telle l'agénésie partielle nécessite une plastie qui doit être réalisée par une équipe multidisciplinaire, spécialisée dans la prise en charge de cette pathologie pour éviter les complications et les récidives et pour ne pas compromettre la sexualité et la fertilité ultérieures de ses jeunes filles.

Dans notre cas, la patiente avait eu une plastie vaginale réalisée par voie combinée vulvaire et abdominale, permettant le drainage de l'hématocolpos. L'intervention était terminée par une réfection du méat urétral corrigeant sa béance iatrogène.

\section{Conclusion:-}

L'hématocolpos est une affection bénigne qui n'affecte la jeune fille qu'à sa puberté. Il est parfois le symptôme révélateur de malformations utérines et rénales. Les conséquences sur la fécondité par le risque d'endométriose ou d'infection ne sont pas à négliger. La prévention passe par un examen soigneux des organes génitaux des nouveauxnés. La prise en charge des hématocolpos secondaires aux malformations vaginales nécessite des centres spécialisés 
pour prévenir les complications et les récidives et ne pas compromettre la sexualité et la fertilité ultérieures de ses jeunes filles.

\section{Conflits d'interet :}

aucun

\section{Iconographie :}

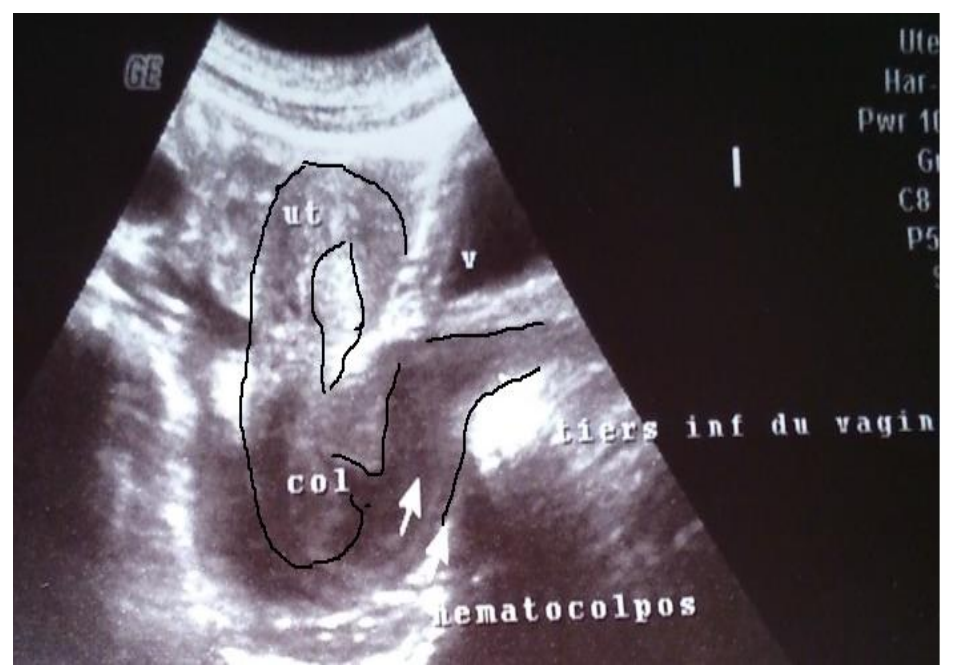

Figure 1:-Coupe longitudinale d'échographie objectivant 2 mois après l'hystérotomie un hématocolpos avec hématométrie

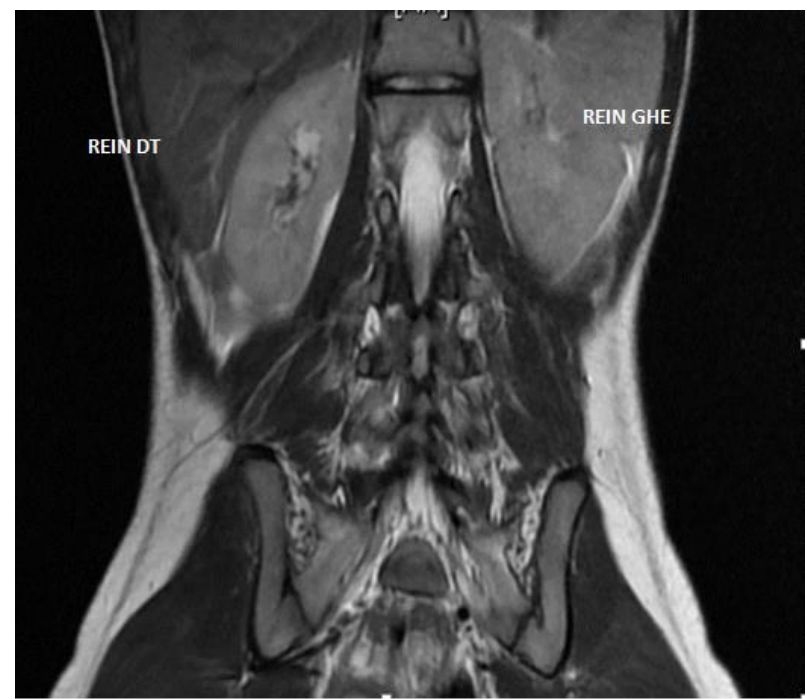

Figure 2:-IRM coupe coronale T2 qui retrouve les 2 reins en place. 


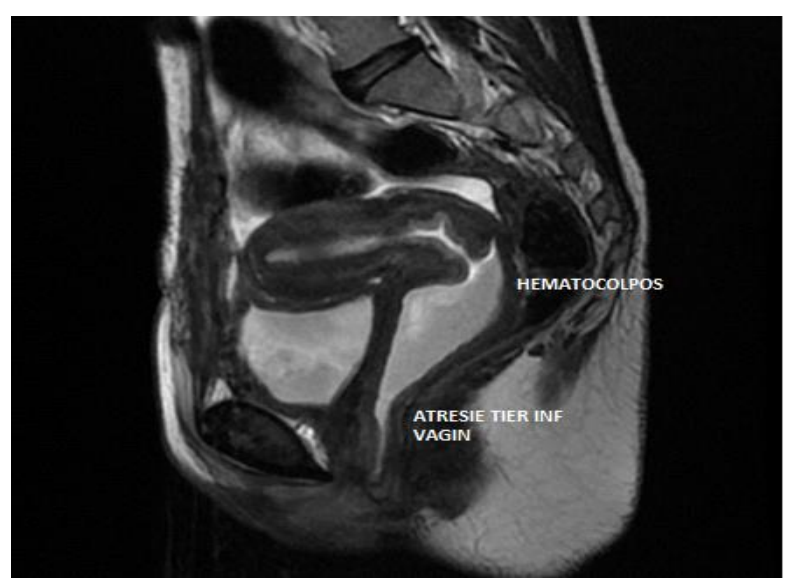

Figure 3:-IRM coupe sagittale qui expose clairement l'utérus d'aspect et de taille normale avec rétention vaginale des $2 / 3$ supérieurs surmontant le tiers inférieur qui est atrésie.

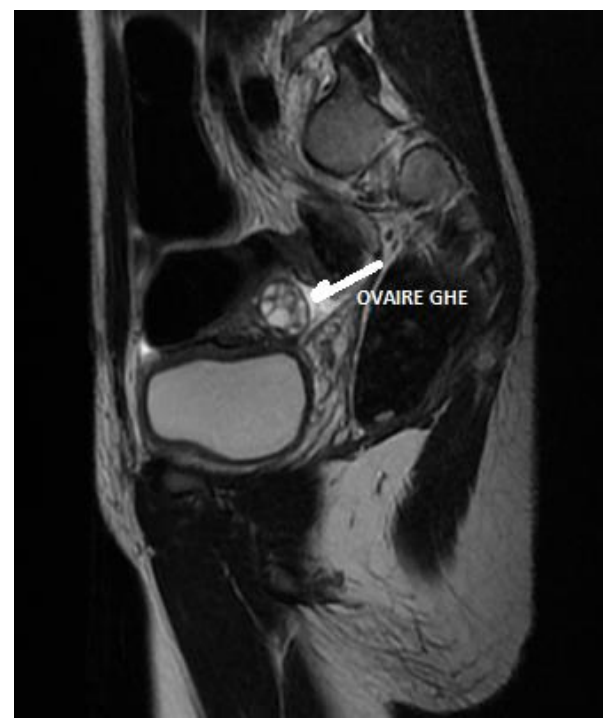

Figure 4:-IRM coupe sagittale T2 montrant la présence del'ovaire gauche d'aspect et de taille normale.

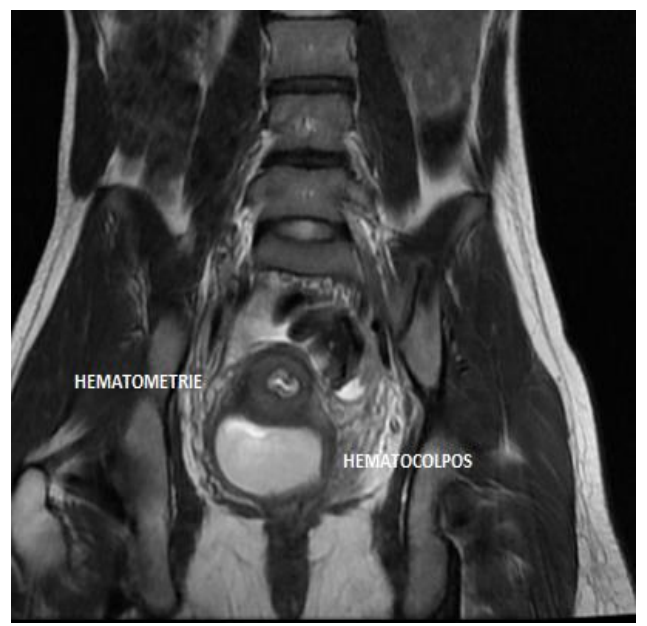

Figure 5:-IRM coupe coronale T2 montrant un hématocolpos surmonté de l'utérus siège d'hématométrie. 


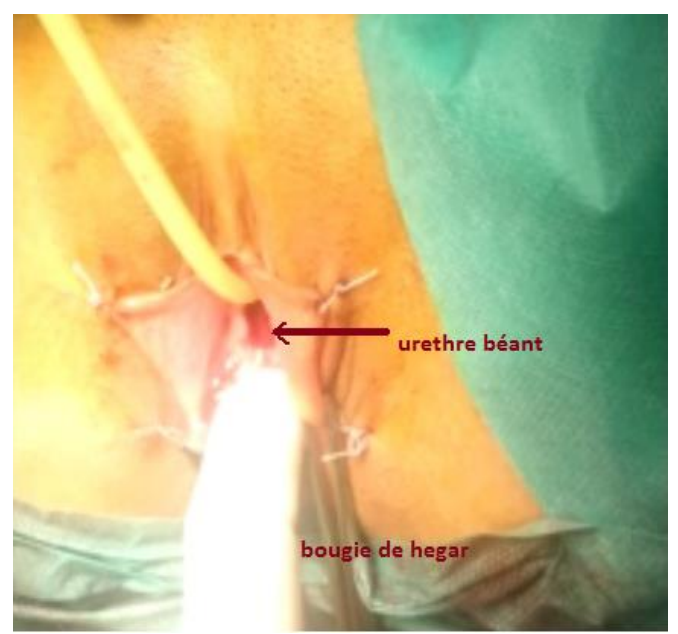

Figure 6:-Urètre béant avec fuite urinaire en dehors de la sonde, le doigt montrant l'absence d'orifice vaginal.

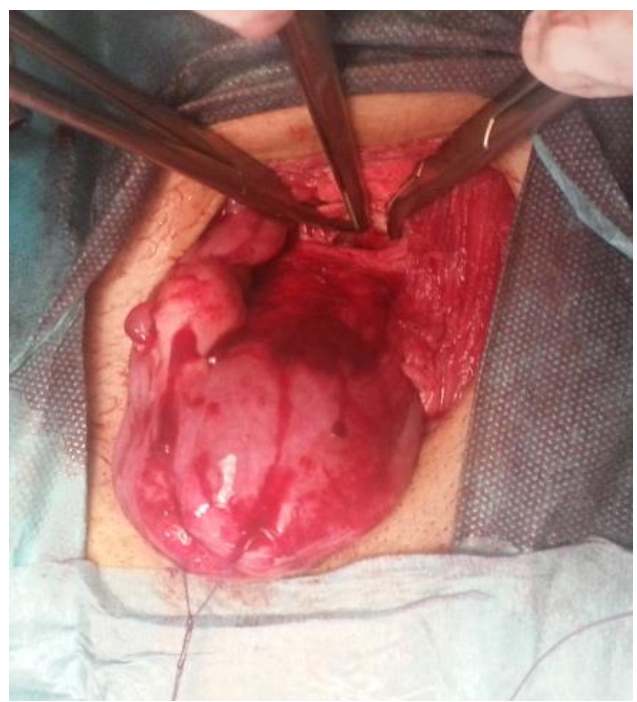

Figure 7:-Ouverture de 1' hématocolpos par abord abdominal, en dessous des utéro sacrés.

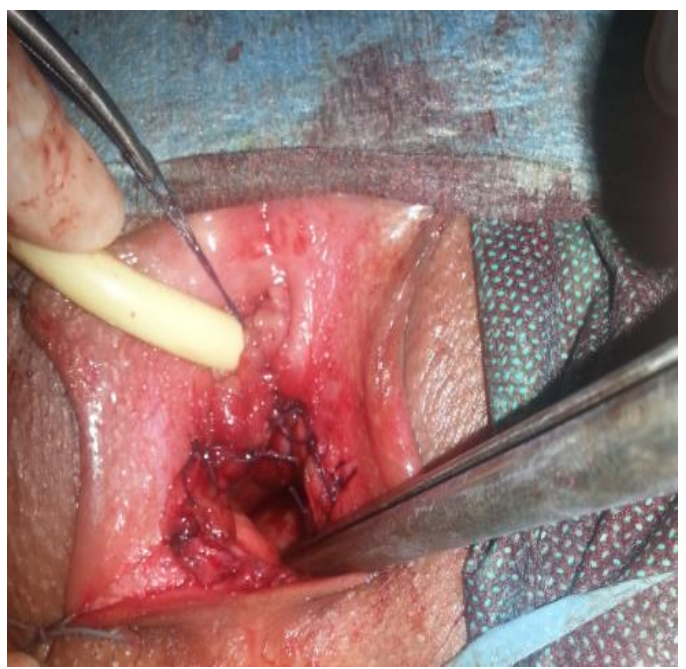

Figure 8:-Points de suture fixant la paroi vaginale à la vulve, créant un néo orifice vulvaire en respectant les orifices de Bartholin. On remarque la correction de la béance urétrale. 


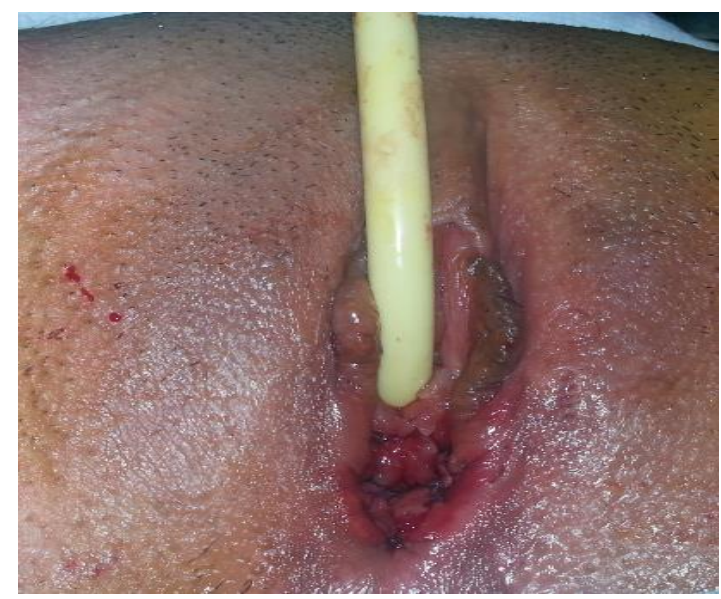

Figure 9:-Résultat final à la fin de l'intervention.

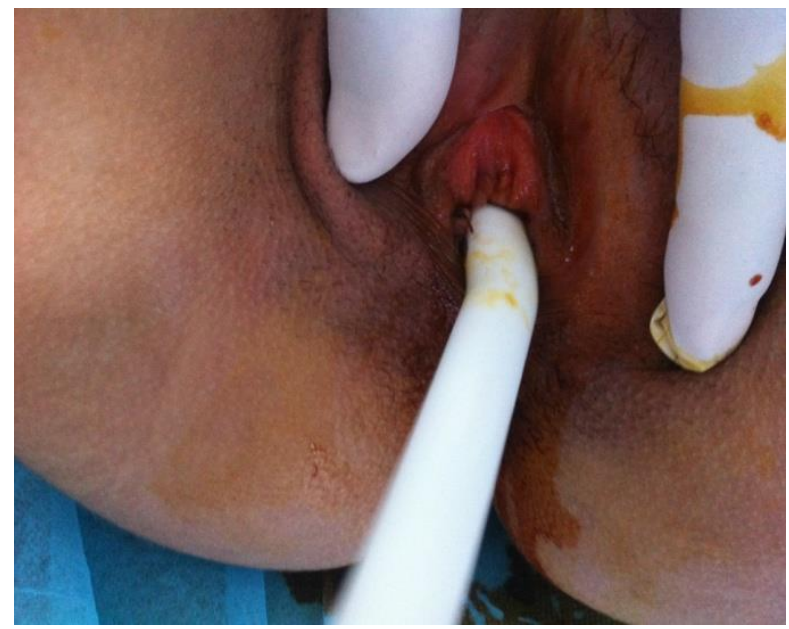

Figure 10:-Introduction d'une bougie de Hégar $\mathrm{n}^{\circ} 12$ le troisième jour post opératoire.

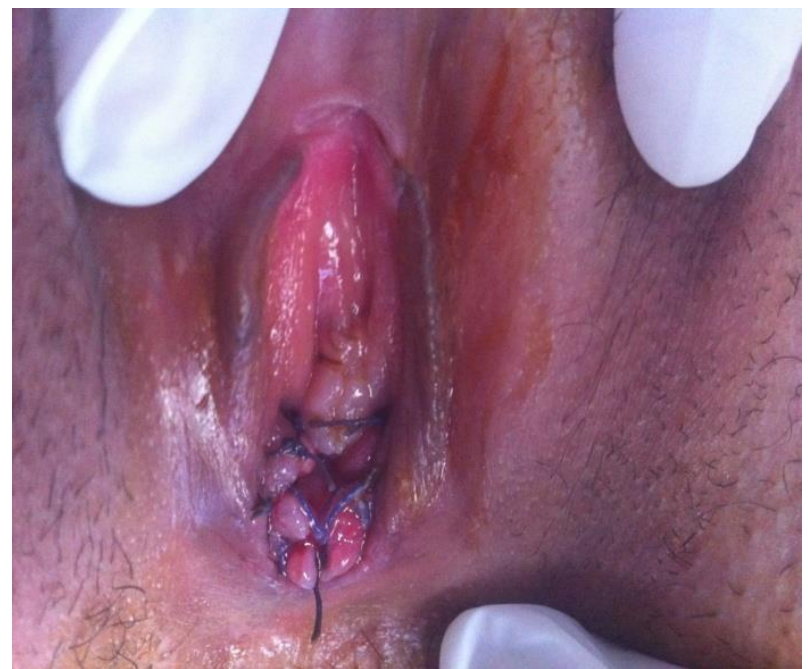

Figure 11:-Résultat au dixième jour post-opératoire, orifice vulvaire perméable avec réduction de la béance du méat urétral

\section{Références:}

1. Fliegner JR, Pepperell RJ. Management of vaginal agenesis with a functioning uterus. Is hysterectomy advisable ? Aust N Z J Obstet Gynecol 1994; 34: 467-70. 
2. Tran AT, Arensman RM, Falterman KW. Diagnosis and management of hydrohematocolpos syndromes. Am J Dis Child 1987; 141: 632-4.

3. Usta IM, Awwad JT, Usta JA, Makarem MM, KaramKS Imperforate hymen: report of an unusual familial occurrence. ObstetGynecol 1993; 82: 655-6.

4. Sanders RM, Nakajima ST. An unusual late presentation of imperforate hymen. Obstet Gynecol 1994; 83: 8968.

5. Randriana B, Irles M, Albert B, Casenave C, Baron JF, Nottin P, Paz CalvezR. Hématocolpos unilatéral, vagin borgne et agénésie de l'arbre urinaire homolatéral.Rev Fr Gynecol Obstet 1994; 89: 401-5.

6. Lafay C, Vernier A, Harajli S. Une curieuse appendicite : hémopéritoine par hématocolpos et hématométrie. J Chir 1993; $130: 105$.

7. Letts M, Haasbeek J. Hematocolpos as a cause of back pain In pre menarchal adolescents. J Pediatr Orthop 1990; 10: 731-2.

8. London NJ, Sefton GK. Hematocolpos. An unusual cause of sciatica in an adolescent girl. Spine 1996; 21: 1381-2.

9. Little HK, Crawford DB, Meister K. Hematocolpos diagnosis made by ultrasound.JCU (J Clin Ultrasound) 1978; 6: 341-2.

10. Van Bommel PJ, Abdullo O. Imperforated hymen as a cause of urinary obstruction.Trop Doct 1996; 26: 133.

11. Wort SJ, Heman-Ackah C, Davies A. Acute urinay retention in the young female. Br J Urol 1995; 76: 667.

12. Yu TJ, Lin MC. Acute urinary retention in two patients with imperforate hymen.Scand J Urol Nephrol 1993; 27: 543-4.

13. Nisanian AC. Hematocolpometra presenting as a urinary retention. A case report..J Reprod Med 1993; 38 : 57 60.

14. Loscalzo IL, Catapano M, Loscalzo J, Sama A. Imperforate hymen with bilateral hydronephrosis : an unusual emergency department diagnosis. J Emerg Med 1995; 13: 337-9.

15. Sanfilippo J, Wakim NG, Schickler KN, Yussman MA. Endometriosis in association with uterine anomaly. Am J Obstet Gynecol 1986; 154: 39-43.

16. Amortegui AJ, Kanbour AI, Silverstein A. Diffuse vaginal adenosis associated with imperforate hymen. Obstet Gynecol 1979; 53: 760-2.

17. Criscuolo JL. Hématocolpos compliqué sur utérus bicorne, bicervical et Adénose vaginale. A propos d'un cas diagnostiqué en urgence devant un hématocolpos surinfecté. Gynecologie 1987; 38: 219-222.

18. Cook S, Hill B. Vaginal carcinoma associated with hematocolpos: a case report and review of vaginal adenosis as a precursor lesion. Aust NZ Obstet Gynecol1995; 35: 465-7.

19. Wang J, Ezat W, Davidson M. Transverse vaginal septum. J Reprod Med 1995; 40: 163-6.

20. Gangopadhyay AN, Pandit SK, Gopal SC. Anorectal anomaly (low) with imperforate hymen in a newborn. Ind Ped 1992; $29: 626-7$.

21. Li YW, Sheih CP, Chen WJ. Unilateral occlusion of duplicated uterus with ipsilateral renal anomaly in young girls: a study with MRI. Ped Rad 1995; 25 suppl: 54-9.

22. Shibata T, Nonomura K, Kakizaki H, Murayama M, Seki T, Koyanagi T. A case of unique communication between blind-ending ectopic ureter and ipsilateral hemi-hematocolpometra in uterus didelphys. J Urol 1995; 153: 1208-10.

23. Carricaburu E, Lewin F, Baron JM, Helardot PG, Sapin E. Kystes de l'ovaire de diagnostic prénatal. Prise en charge prénatale. Med foet écho Gynecol 1997; 30: 17-21.

24. Riboni G, De Simoni MM, Serantoni S, Copalini B, Villa G. Un caso di idrometrocolpoos servato ecograficamente in epoca prenatale. Radiol Med 1989; 77: 2857.

25. Winderl LM, Silverman RK. Prenatal diagnosis of congenital imperforate hymen.Obstet Gynecol 1995; 85: $857-60$. 